\title{
Alendronate Enhances Functional Recovery after Spinal Cord Injury
}

\author{
Yuna Choi* and Taekyun Shin* \\ Department of Veterinary Anatomy, College of Veterinary Medicine and Veterinary Medical Research Institute, \\ Jeju National University, Jeju 63243, Korea
}

\begin{abstract}
Spinal cord injury is a destructive disease characterized by motor/sensory dysfunction and severe inflammation. Alendronate is an anti-inflammatory molecule and may therefore be of benefit in the treatment of the inflammation associated with spinal cord injury. This study aimed to evaluate whether alendronate attenuates motor/sensory dysfunction and the inflammatory response in a thoracic spinal cord clip injury model. Alendronate was intraperitoneally administered at $1 \mathrm{mg} / \mathrm{kg} /$ day or $5 \mathrm{mg} / \mathrm{kg} /$ day from day (D) 0 to 28 post-injury (PI). The histopathological evaluation showed an alleviation of the inflammatory response, including the infiltration of inflammatory cells, and a decrease in gliosis. Alendronate also led to reductions in the levels of inflammation-related molecules, including mitogen-activated protein kinase, p53, pro-inflammatory cytokines, and pro-inflammatory mediators. Neuro-behavioral assessments, including the Basso, Beattie, and Bresnahan scale for locomotor function, the von Frey filament test, the hot plate test, and the cold stimulation test for sensory function, and the horizontal ladder test for sensorimotor function improved significantly in the alendronate-treated group at D28PI. Taken together, these results suggest that alendronate treatment can inhibit the inflammatory response in spinal cord injury thus improving functional responses.
\end{abstract}

Key words: Alendronate, Behavioral test, Gliosis, Inflammation, Spinal cord injury

\section{INTRODUCTION}

Spinal cord injury (SCI), including contusion, transection and compression, is accompanied by secondary degeneration, autonomic dysfunction and an inflammatory response [1]. The location and severity of the paralysis resulting from SCI differs by site [2]. Along with the hind-limb paralysis after the thoracic SCI, the SCI rats were suffered from processing the sensory cognition, such as hyperalgesia or insensibility [3]. The behavioral disability of SCI rats was estimated in a variety of behavioral assays, including the Basso, Beattie and Bresnahan score for locomotor function [4], the

Submitted September 9, 2021, Revised December 12, 2021,

Accepted January 12,2022

* To whom correspondence should be addressed.

Taekyun Shin, TEL: 82-64-754-3363, FAX: 82-64-756-3354

e-mail:shint@jejunu.ac.kr

Yuna Choi, TEL: 82-64-754-3363, FAX: 82-64-756-3354

e-mail:cyn323@jejunu.ac.kr von Frey filament test for mechano-sensory function [5], the hot [6] or cold stimulation test [7] for thermo-sensory function and the horizontal ladder walking test for sensorimotor function [8]. As a severe inflammatory response after the spinal cord injury, the infiltration of inflammatory cells, microgliosis, and astrogliosis [1, 9] was emerged. The infiltration of immune cells was composed of mainly neutrophil in the early stage of SCI and turned to be replaced with blood-borne monocyte, especially macrophages in the later stage of SCI [3]. The glial activation, including microglia and astroglia was also dominated after the SCI and was contributed to release the pro-inflammatory cytokines and chemokines, representatively interleukin-1 beta (IL-1 $\beta$ ) and tumor necrosis factoralpha (TNF-a) [10]. Mitogen-activated protein kinase (MAPK) signaling, such as extracellular signal-regulated kinase (ERK), cjun-NH2-terminal kinase (JNK) and p38, is activated after the SCI and activation occurs in parallel with the stimulation of pro-inflammatory cytokines [11]. Among the therapeutic targets of SCI are a reduction of the secondary damage, stem cell replacement
Copyright (c) Experimental Neurobiology 2022. www.enjournal.org
This is an Open Access article distributed under the terms of the Creative Commons Attribution Non-Commercial License (http://creativecommons.org/licenses/by-nc/4.0) which permits unrestricted non-commercial use, distribution, and reproduction in any medium, provided the original work is properly cited. 
therapy, the removal of inhibitory molecules, promotion of the regenerative response, and improvement of axonal growth, remyelination, and rehabilitation [12]. The most common therapeutic target of SCI is a reduction of secondary damage using neuroprotective or anti-inflammatory molecules.

Alendronate is a nitrogen-containing bisphosphonate that can cross the blood-brain barrier [13] and is widely used to treat osteoporosis [14]. Alendronate regulates farnesyl pyrophosphate and geranylgeranyl pyrophosphate synthesis in the mevalonate pathway [15]. Some recent clinical trials suggested that alendronate relieves pain, non-steroidal anti-inflammatory drug resistance, and stiffness, and improves the movement of affected joints [16]. The anti-inflammatory effect of alendronate, demonstrated in experimental autoimmune encephalomyelitis, an animal model of human multiple sclerosis, occurs through a decrease in infiltration of inflammatory cells and pro-inflammatory cytokines, including IL-1 $\beta$, TNF- $\alpha$, and interferon-gamma [17]. The serine protease inhibitor, clade A, member $3 \mathrm{~N}$ (sepina3n) is highly found in brain, testis and spleen [18]. The physiological function of serpina $3 \mathrm{n}$ is involved in various pathologies, including the complement cascade, apoptosis and wound healing [19]. Serpina3n, as a protease inhibitor, inactivate anti-chymotrypsin, cathespin G, granzyme B and leukocyte elastase [19]. Another significant role of serpina3n is known as a novel marker of reactive astrocyte during central nervous system insults and overexpressed serpina $3 \mathrm{n}$ is detected under Alzheimer [20], hypothalamus inflammation [21] and ischemic stroke [21]. However, the relationship between alendronate and pro-inflammatory mediators requires further research in central nervous system diseases, including SCI.

Thus, this study evaluated the effect of alendronate on SCI, and the underlying mechanism based on histopathological and behavioral test.

\section{MATERIALS AND METHODS}

\section{Animals}

Female Sprague-Dawley rats (6 7 weeks old; Samtako, Gyeonggi-do, Korea) were maintained in our facility under normal conditions $\left(12\right.$-h light $/$ dark cycle, temperature $=23 \pm 2^{\circ} \mathrm{C}$ ). All animal protocols conformed to international laws and National Institutes of Health policies, including the Care and Use of Laboratory Animals (National Institutes of Health publication no. 85-23, 1985, revised 1996). All experimental procedures were performed according to the Guidelines for the Care and Use of Laboratory Animals of Jeju National University (permission number: 2021-0009).

\section{Experimental groups and procedure}

Clip compression injury was induced as in our previous studies [22]. Briefly, the rats were anesthetized with Zoletil ${ }^{\circledR} 50(0.2 \mathrm{ml} /$ animal, Virbac, Carros, France) and laminectomized at the spinous process of the tenth thoracic vertebra. The exposed thoracic spinal cord was compressed horizontally using a 35 55 g vascular clip (00466; S\&T ${ }^{\circledR}$, Rheinfall, Switzerland) for $1 \mathrm{~min}$. Thereafter, the surgical site, including the muscle and skin layers, were sutured. The rats were classified into five groups to investigate the effect of alendronate on SCI: normal control $(n=10)$, sham control ( $n=10$ per sampling date), SCI+vehicle $(n=10$ per sampling date), SCI+alendronate $1 \mathrm{mg} / \mathrm{kg}$ ( $\mathrm{n}=10$ per sampling date) and $\mathrm{SCI}+$ alendronate $5 \mathrm{mg} / \mathrm{kg}$ ( $\mathrm{n}=10$ per sampling date). Alendronate was administrated intraperioneally to SCI rats from day (D) 0 post-injury (PI) to D28PI (Y0001727; $1 \mathrm{mg} / \mathrm{kg} /$ day or $5 \mathrm{mg} / \mathrm{kg} /$ day; EDQM, Strasbourg, France) [23].

\section{Tissue preparation}

The rats were killed with 95\% $\mathrm{CO}_{2}$ gas on D3.5PI, D7PI, and D28PI. Thoracic spinal cord samples (T9 T11), including approximately $0.5 \mathrm{~cm}$ back and forth of the epicenter, were obtained and fixed in $4 \%$ paraformaldehyde for histopathological examination. The samples were stored at $-80^{\circ} \mathrm{C}$ until western blotting and real time-polymerase chain reaction (RT-PCR) analysis.

\section{Histological examination}

The fixed tissues were embedded in paraffin wax and sectioned to 5 - $\mu$ m-thickness with a microtome (RM 2135; Leica, Nussloch, Germany). After deparaffinization with xylene and 100\% 70\% of ethanol, the sections were stained with hematoxylin and eosin.

\section{Immunohistochemistry}

The same immunohistochemistry protocol used in our previous study was performed [24]. In brief, the sections were incubated with the primary antibodies listed in Table 1 for $1 \mathrm{~h}$ at room temperature. The matched secondary antibodies were reacted to the slides based on the primary antibody. An avidin-biotin complex kit (Vectastain Elite ABC Kit; Vector Laboratories) was applied. A 3-3'-diaminobenzidine substrate kit (Vector Laboratories, Burlingame, CA, USA) was used to visualize the peroxidase reaction, and the sections were counterstained with hematoxylin. The stained slides were captured and analyzed with the optical microscope (BX51; Olympus, Japan).

\section{Western blot analysis}

The western blot analysis proceeded according to our previous studies [24]. The primary antibodies are listed in Table 1. The 
Table 1. The characterization of antibodies

\begin{tabular}{|c|c|c|c|}
\hline Antigen & Immunogen & Manufacturer, species, antibody type & Dilution \\
\hline$\beta$-actin & $\begin{array}{l}\text { Synthetic } \beta \text {-cytoplasmic actin N-terminal peptide conjugated to } \\
\text { KLH }\end{array}$ & Sigma Aldrich (a5441), mouse, monoclonal & $1: 10,000$ \\
\hline ED1 & Rat spleen cells & Bio-rad (MCA341), mouse, monoclonal & $1: 800$ \\
\hline ERK1/2 & $\begin{array}{l}\text { Synthetic peptide corresponding to residues near the C-terminus } \\
\text { of human p44 MAP kinase }\end{array}$ & $\begin{array}{l}\text { Cell Signaling Technology (\#4695), rabbit, } \\
\text { monoclonal }\end{array}$ & $1: 1,000$ \\
\hline GFAP & Purified GFAP from pig spinal cord was used as the immunogen & Sigma-Aldrich (G3893), mouse, monoclonal & $1: 1,000$ \\
\hline Ibal & Synthetic peptide of the $\mathrm{C}$ terminal of Ibal & WAKO (019-19741), rabbit, polyclonal & $1: 1,000$ \\
\hline JNK1/2 & Recombinant human JNK2 fusion protein & Cell Signaling Technology (\#9252), rabbit, polyclonal & $1: 1,000$ \\
\hline p38 & Peptide mapping at the $\mathrm{C}$-terminus of $\mathrm{p} 38 \mathrm{a}$ of mouse origin. & Santa Cruz Biotechnology (sc-535), rabbit, polyclonal & $1: 1,000$ \\
\hline p53 & Amino acids 376-378 of human p53 & Calbiochem (\#OP03L), mouse, monoclonal & $1: 1,000$ \\
\hline $\mathrm{p}$-ERK1/2 & $\begin{array}{l}\text { Synthetic phosphopeptide corresponding to residues surrounding } \\
\text { Thr202/Tyr204 of human p } 44 \text { MAP kinase }\end{array}$ & $\begin{array}{l}\text { Cell Signaling Technology (\#4370), rabbit, } \\
\text { monoclonal }\end{array}$ & $1: 1,000$ \\
\hline $\mathrm{p}-\mathrm{JNK} 1 / 2$ & $\begin{array}{l}\text { Synthetic phosphopeptide corresponding to residues surrounding } \\
\text { Thr183/Tyr } 185 \text { of human SAPK/JNK }\end{array}$ & Cell Signaling Technology (\#9251), rabbit, polyclonal & $1: 1,000$ \\
\hline p-p38 & Tyr 182 phosphorylated p38a MAPK of human origin. & $\begin{array}{l}\text { Santa Cruz Biotechnology (sc-7973), mouse, } \\
\text { polyclonal }\end{array}$ & $1: 1,000$ \\
\hline
\end{tabular}

ERK1/2, extracellular signal-regulated kinase1/2; GFAP, glial fibrillary acidic protein; Ibal, ionized calcium-binding adapter molecule 1; JNK1/2, c-junNH2-terminal kinase1/2; p-ERK1/2, phosphorylated form of extracellular signal-regulated kinase1/2; p-JNK1/2, phosphorylated form of c-jun-NH2terminal kinase1/2; p-p38, phosphorylated form of p38.

Table 2. The primer sequences

\begin{tabular}{lll}
\hline \multicolumn{1}{c}{ Primers } & \multicolumn{1}{c}{ Forward sequence } & \multicolumn{1}{c}{ Reverse sequence } \\
\hline COX-2 & 5'-CGG AGG AGA AGT GGG GTT TA-3' & 5'-TGG GAG GCA CTT GCG TTC AT-3' \\
GAPDH & 5'-GGG GGC TCT CTG CTC CTC CC-3' & 5'-CGG CCA AAT CCG TTC ACA CCG-3' \\
IL-1 $\beta$ & 5'-CCC TGC AGC TGG AGA GTG TGG-3' & 5'-TGT GCT CTG CTT GAG AGG TGC-3' \\
TNF- $\alpha$ & 5'-CGT CGT AGCAAA CCA CCA AG-3' & 5'-CACAGA GCA ATG ACT CCA AA-3' \\
\hline
\end{tabular}

COX-2, cyclooxygenase-2; GAPDH, glyceraldehyde-3-phosphate dehydrogenase; IL- $1 \beta$, interleukin- 1 beta; TNF- $\alpha$, tumor necrosis factor-alpha.

primary antibody was reacted with horseradish peroxidase antimouse IgG (PI-2000, Vector Labs, Burlingame, CA, USA) or antirabbit IgG (PI-1000, Vector Labs, Burlingame, CA, USA) on the membrane overnight at $4^{\circ} \mathrm{C}$. Enhanced chemiluminescence substrate (BS ECL Plus Kit, W6002; Biosesang, Gyeonggi, Korea) was used to visualize the immunoblot signals, which were quantified using Solo 6X and FUSION software (Vilber Lourmat, Collégien, France). The values of each immunoblot were normalized to $\beta$-actin.

\section{Real time PCR}

Total RNA of the spinal cord in all groups ( $\mathrm{n}=6$ per group) at D7PI was extracted using TRIzol RNA Isolation Reagent (Life Technologies, Carlsbad, CA, USA). The cDNA was transcribed from purified RNA using the $5 \times$ First Strand cDNA Synthesis Master Mix (CellSafe, Gyeonggi-do, Korea). The PCR was performed with the minimum inhibitory concentration using the mic instrument (Bio Molecular Systems, Potts Point, Australia) and $2 \times$ Quantity SYBR Green (PhileKorea Co., Ltd., Seoul, Korea) as follows: 55 cycles of denaturation $\left(10 \mathrm{~s}, 95^{\circ} \mathrm{C}\right)$, annealing $(10 \mathrm{~s}$, $60^{\circ} \mathrm{C}$ ), and extension $\left(10 \mathrm{~s}, 72^{\circ} \mathrm{C}\right.$ ). The Serpina3n primer (qRno-
CID0005765, BioRad, CA, USA) and other primer sequences are summarized in Table 2.

\section{Behavioral test}

Locomotor function after SCI was evaluated using the Basso, Beattie, and Bresnahan rating scale [4]. Briefly, the Basso, Beattie and Bresnahan rating scale was categorized the behavioral abnormality with the coordination of gait and the movable joints into 22 grades from 0 to 21 based on the severity of the injured lesion. The lower grade represented the more incomplete locomotor function because of severe damaged lesion, whereas the higher grade signified the recovery of the locomotor function or the lesser damaged lesion. Sensory function was evaluated using the von Frey filament test [5], in which a positive or negative response depends on the filament force. The results were calculated as the 50\% threshold gram according to the following equation:

$$
10^{\mathrm{Xf}+\kappa \delta} / 10,000
$$

Where Xf, value (in log units) is the final von Frey filament used, $\kappa$, is the tabular value of the pattern of positive/negative responses, 
and $\delta$ is mean difference (in log units) between stimuli.

The hot plate test [6], and the cold stimulation test using acetone [7] were used to measure the latency of hind limb avoidance to hot or cold stimuli. Sensorimotor function was comprehensively evaluated in a horizontal ladder test $[8]$ and rated from 0 to $6(0$, total miss; 1 , deep slip; 2 , slight slip; 3 , replacement; 4, correction; 5, partial placement; and 6 , correct placement). The behavioral tests were evaluated in a double-blinded manner until D28PI. Spinal cord samples were obtained after the behavioral tests.

\section{Statistical analysis}

All values analyzed were mean \pm standard error of three independent experiments. The results were analyzed using one-way analysis of variance followed by two-way ANOVA test for multiple comparisons. A p-value $<0.05$ was considered significant. Three different sections from each rat were analyzed under the three blind manner and the percentage of stained area [(positive area/ total area) $\times 100(\%)]$ was calculated. The positive area was measured by the Image J software (NIH, Bethesda, MD, USA).

\section{RESULTS}

\section{Alendronate improved locomotor and sensorimotor function}

The Basso, Beattie, and Bresnahan score was calculated daily from D0PI. The SCI rats exhibited significant deficits in locomotor function during the experiment. However, recovery of locomotor function was confirmed by an increase in the score from D10PI in the SCI+alendronate $1 \mathrm{mg} / \mathrm{kg}$ and $5 \mathrm{mg} / \mathrm{kg}$-treated group. The score tended to increase until D28PI in the SCI+alendronate $1 \mathrm{mg} /$ $\mathrm{kg}$ and $5 \mathrm{mg} / \mathrm{kg}$-treated group, relative to that of the SCI+vehicletreated group (Fig. 1A). Sensory, including thermal and mechani$\mathrm{cal}$, and sensorimotor function were tested before sampling. The hot plate and cold stimulation tests were used to examine thermal sensitivity (Fig. 1B and 1C). A loss of temperature sensitivity was detected at D3.5PI and D7PI in the SCI rats. Significant deficits remained in the SCI+vehicle-treated group on D28PI, whereas the latency of recognition of hot and cold had recovered in the $\mathrm{SCI}+$ alendronate $1 \mathrm{mg} / \mathrm{kg}$ and $5 \mathrm{mg} / \mathrm{kg}$-treated group. The von Frey filaments of varying thickness were used to assess the sensitivity to mechanical stimuli (Fig. 1D). The SCI rats showed significantly less responsivity, compared to the normal and sham control until the end of the experiment. However, the SCI+alendronate $1 \mathrm{mg} / \mathrm{kg}$ and $5 \mathrm{mg} / \mathrm{kg}$-treated group showed higher sensitivity in terms of the $50 \%$ paw withdrawal threshold than the SCI+vehicletreated group. The horizontal ladder test was carried out preSCI and on the sampling date (Fig. 1E). Pre-SCI, the rats received high scores. The SCI rats, which were paralyzed in the hind limbs, were unable to cross the horizontal ladder without error, while the SCI+alendronate $1 \mathrm{mg} / \mathrm{kg}$ and $5 \mathrm{mg} / \mathrm{kg}$-treated group had better gait performance than the SCI+vehicle-treated group. The significant difference between alendronate $1 \mathrm{mg} / \mathrm{kg}$ and $5 \mathrm{mg} / \mathrm{kg}$ was not observed so only the SCI+alendronate $1 \mathrm{mg} / \mathrm{kg}$-treated group was evaluated in other analysis. The alendronate treatment was involved in the improvement of behavioral disability including motor function, sensory function and locomotor function, and these results mean that the alendronate is a potential candidate to relieve the behavioral malfunction after the SCI. Therefore, the possible pathway of the therapeutic effect of alendronate was proved by following experiment.

\section{Histopathological examination}

The histopathological examination was performed using the normal control, the SCI+vehicle-treated group, and the $\mathrm{SCI}+$ alendronate $1 \mathrm{mg} / \mathrm{kg}$-treated group at D7PI. In the core region of the spinal cords of the SCI-induced rats (Fig. 2B and 2C), hemorrhage, vacuolar degeneration and infiltration of inflammatory cells were observed, but in the normal control, there were no infiltrated cells (Fig. 2A). A positive immunoreaction to the macrophage marker ED1 was also not detected in the normal control (Fig. 2D), whereas an intense reaction was detected in both the SCI+vehicle-treated group and the SCI+alendronate $1 \mathrm{mg} / \mathrm{kg}$ treated groups (Fig. 2E and 2F). However, the ED1-positive area in $\mathrm{SCI}+$ alendronate $1 \mathrm{mg} / \mathrm{kg}$-treated group was significantly smaller than that of the SCI+vehicle-treated group (Fig. 2G). This result implicated that alendronate had an effect on suppression of ED1positive macrophage infiltration. The declined inflammatory cell infiltration is expected to refer to make the pro-inflammatory cytokines and mediators to be dwindled.

\section{Alendronate reduced the activation of microglia and astrocytes}

The activation of microglia and astrocytes was investigated immunohistochemically using antibodies against ionized calciumbinding adapter molecule 1 (Ibal) and glial fibrillary acidic protein (GFAP). An Ibal-positive immunoreaction was detected in the normal control (Fig. 3A) and in the SCI-induced rats (Fig. 3B and $3 \mathrm{C}$ ). However, the Ibal-positive area in the SCI+alendronate $1 \mathrm{mg} / \mathrm{kg}$-treated group was significantly smaller than that in the SCI+vehicle-treated group (Fig. 3D). Similarly, a GFAP-positive immunoreaction was detected in the normal control (Fig. 3E) and in SCI-induced rats treated with vehicle (Fig. 3F) or alendronate $1 \mathrm{mg} / \mathrm{kg}$ (Fig. 3G), but the GFAP-positivity was significantly less in the SCI+alendronate $1 \mathrm{mg} / \mathrm{kg}$-treated group than in the 

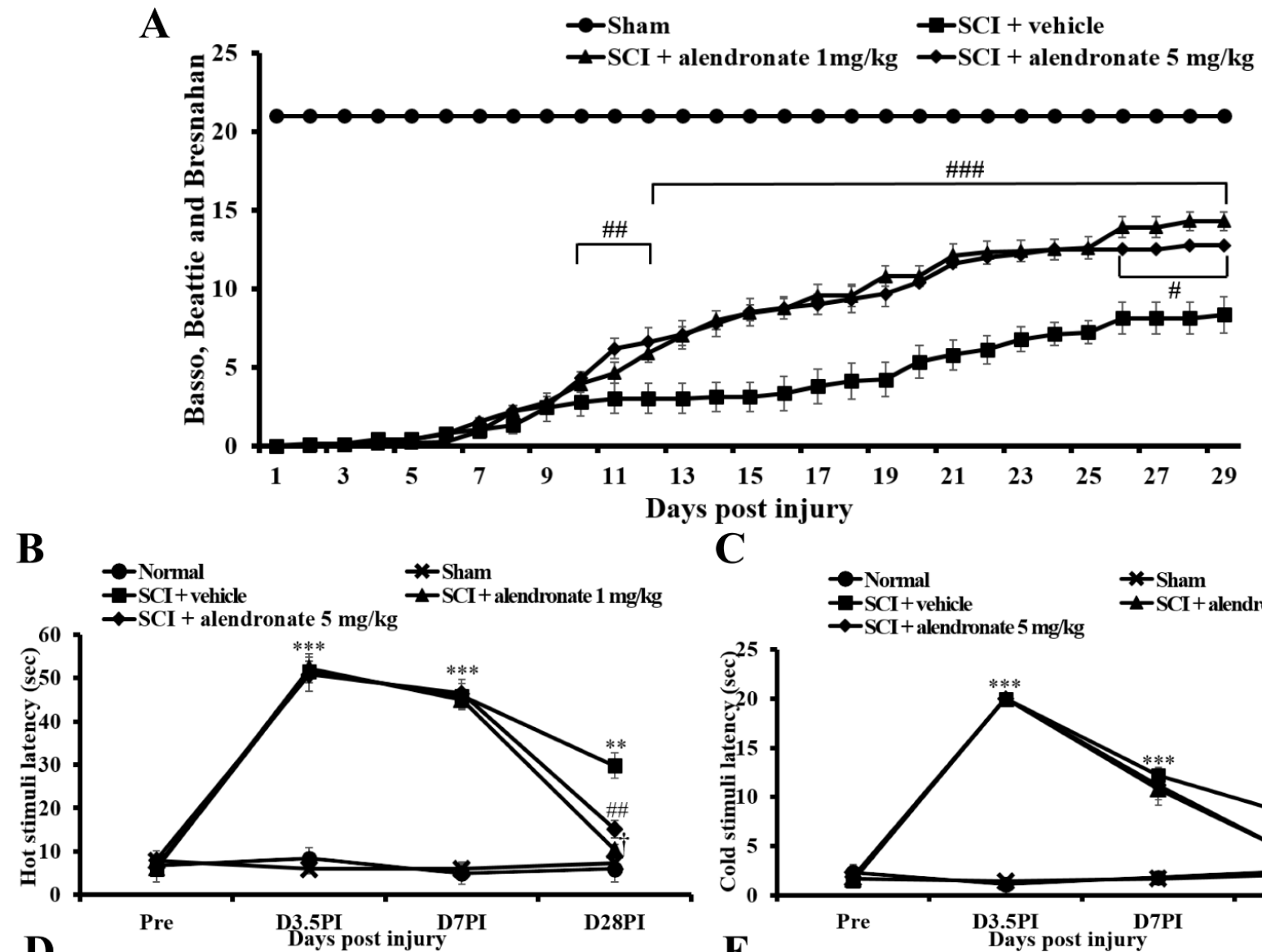

C
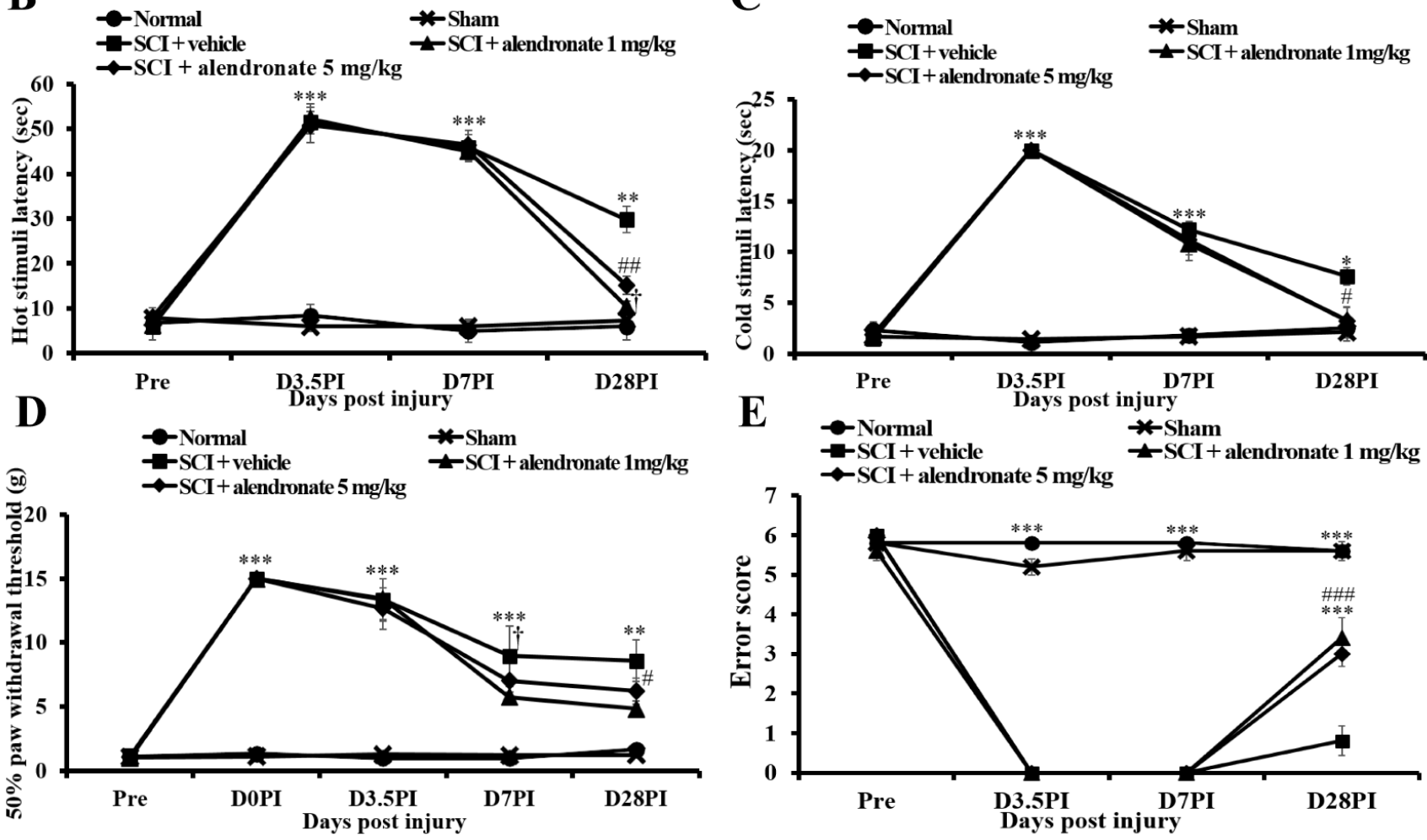

Fig. 1. Behavioral assessment. (A) Basso, Beattie, and Bresnahan score. (B) Hot stimuli test. (C) Cold stimuli test. (D) von Frey filament test. (E) Horizontal ladder walking test. ${ }^{*} \mathrm{p}<0.05 ;{ }^{* *} \mathrm{p}<0.01 ;{ }^{* * *} \mathrm{p}<0.001$ vs. normal control. ${ }^{*} \mathrm{p}<0.05 ;{ }^{* *} \mathrm{p}<0.01 ;{ }^{* \# *} \mathrm{p}<0.001$ vs. spinal cord injury (SCI)+vehicle-treated group. ${ }^{\dagger} \mathrm{p}<0.05 \mathrm{vs}$. SCI+alendronate $1 \mathrm{mg} / \mathrm{kg}$-treated group.

SCI+vehicle-treated group (Fig. 3H). To do nomotopic signal transduction in nervous system, the proper degree of neuron and glial cell interaction was needed and the excessive glial cell activation was led to interrupting the functional recovery. The mitigation of glial cells activation, including Iba1-positive microglia and GFPA-positive astrocyte was able to support the behavioral improvement by alendronate treatment.

\section{Alendronate suppressed the activation of MAPK signaling}

The MAPK signaling, including ERK1/2 (approximately 44 and $42 \mathrm{kDa}$ ), JNK1/2 (approximately 46 and $54 \mathrm{kDa}$ ) and p38 (approximately $38 \mathrm{kDa}$ ), and p53 are indicators of neuro-inflammation and are activated in SCI [22]. At D3.5PI, the ratio of p-ERK2/total ERK2 (2.93 \pm 0.45 fold; $\mathrm{p}<0.05)$ and $\mathrm{p}$-JNK2/total JNK2 $(3.18 \pm 0.78$ fold; $\mathrm{p}<0.05)$ in the $\mathrm{SCI}+$ alendronate $1 \mathrm{mg} / \mathrm{kg}$-treated group 


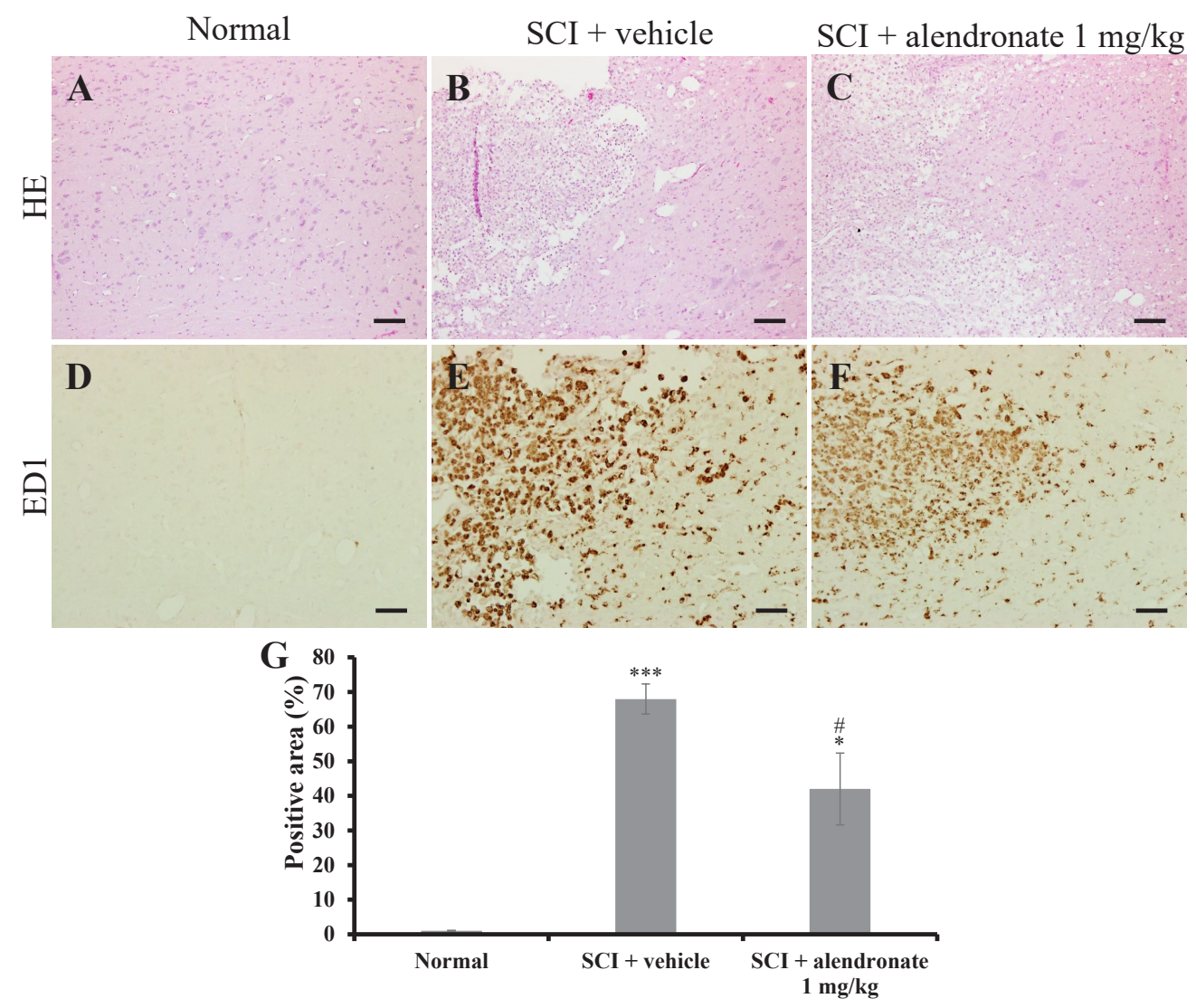

Fig. 2. Histopathological evaluation of the spinal cord in normal control and SCI-induced rats on D7PI. (A C) Cell infiltration in SCI rats (B and C) normal controls (A). ED1-positive macrophages in the core lesions of the SCI+vehicle-treated group (E) and SCI+alendronate $1 \mathrm{mg} / \mathrm{kg}$-treated group (F). Under alendronate treatment, the size of the ED1-positive area was reduced $(\mathrm{G})$. Scale bars $=50 \mu \mathrm{m} .{ }^{*} \mathrm{p}<0.05 ;{ }^{* * *} \mathrm{p}<0.001 \mathrm{vs}$. normal control. ${ }^{*} \mathrm{p}<0.05$ vs. SCI+vehicle-treated group.
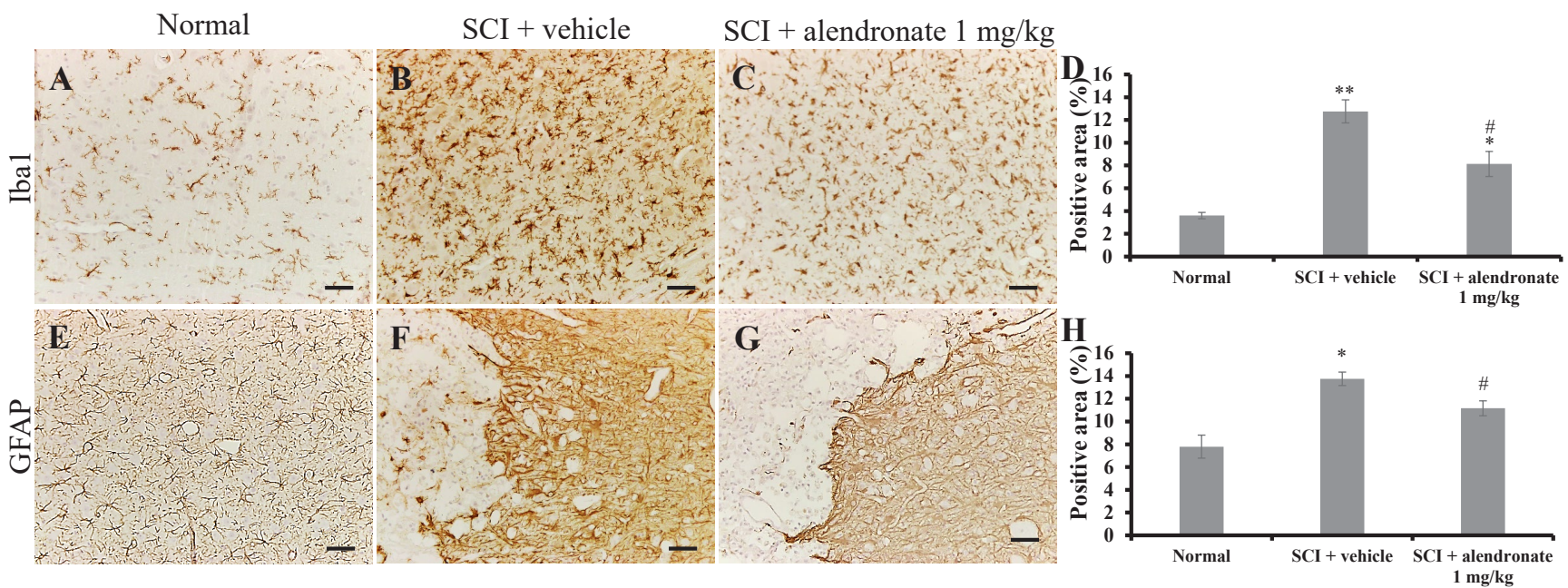

Fig. 3. Immunohistochemical analysis of ionized calcium-binding adapter molecule 1 (Ibal) and glial fibrillary acidic protein (GFAP) in normal control and SCI-induced rats on D7PI. Ibal-positivity in normal (A), SCI+vehicle-treated (B) and SCI+alendronate $1 \mathrm{mg} / \mathrm{kg}$-treated (C) rats. A semi-quantitative analysis showed the reduced activation of microglia in the SCI+alendronate $1 \mathrm{mg} / \mathrm{kg}$-treated group (D). GFAP staining was detected in normal (E), SCI+vehicle-treated (F) and SCI+alendronate $1 \mathrm{mg} / \mathrm{kg}$-treated $(\mathrm{G})$ rats. A semi-quantitative analysis showed a significant decrease in GFAP staining in the SCI+alendronate $1 \mathrm{mg} / \mathrm{kg}$-treated group $(\mathrm{H})$. Scale bars $=50 \mu \mathrm{m} .{ }^{*} \mathrm{p}<0.05 ;{ }^{* *} \mathrm{p}<0.01$ vs. normal control. ${ }^{*} \mathrm{p}<0.05$ vs. SCI+vehicle-treated group. 

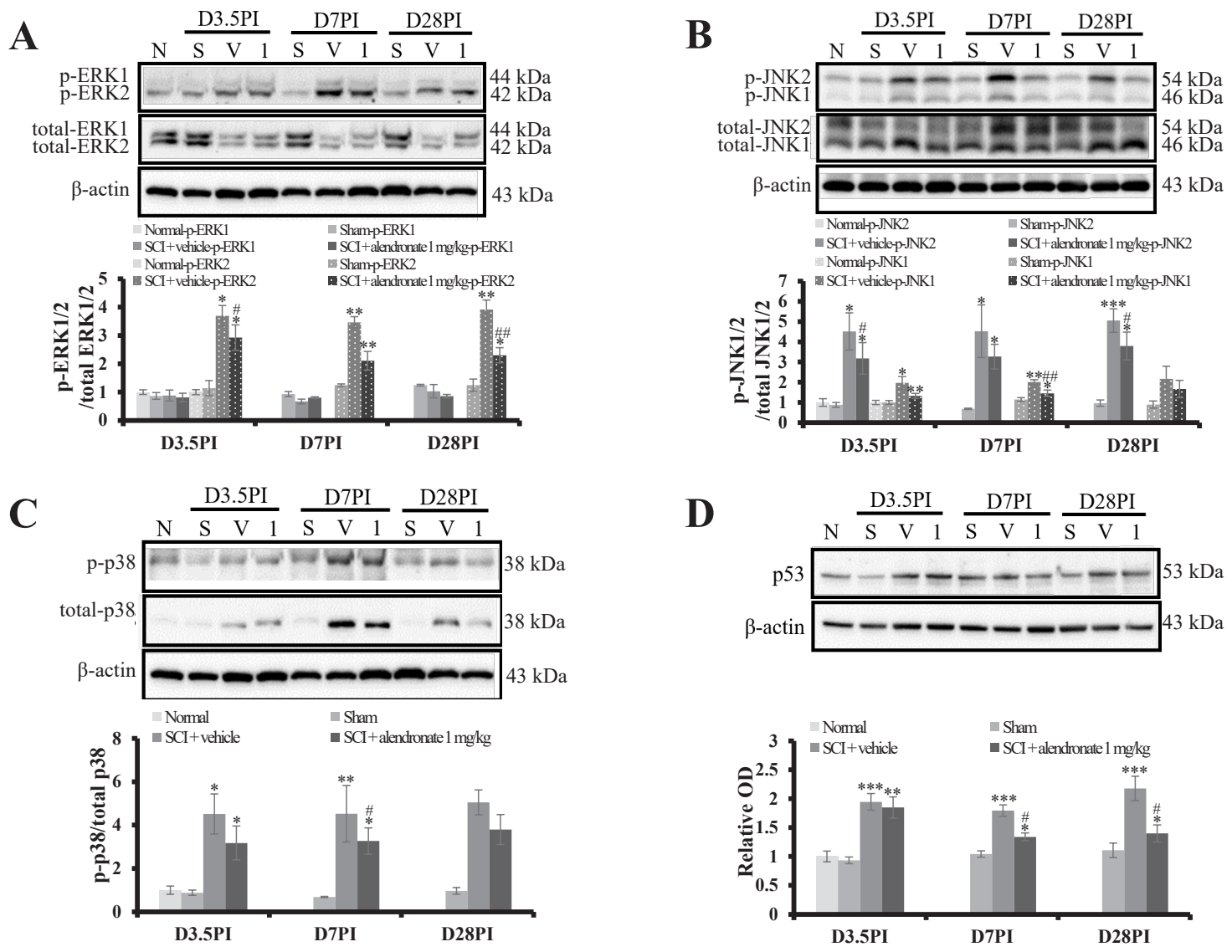

Fig. 4. Western blot of mitogen-activated protein kinase (MAPK) signaling and p53. Representative immunoblots of phosphorylated extracellular signal-regulated kinase1/2 (p-ERK1/2; 44 and $\sim 42 \mathrm{kDa}$ ) (A), c-jun-NH2-terminal kinase1/2 (p-JNK1/2; 46 and 54 kDa) (B), and phosphorylated p38 (p-p38; 38 kDa) (C), and p53 (D). The expression levels of p-ERK2/total ERK2 (A), p-JNK1/2/total JNK1/2 (B), and p-p38/total p38 (C) in the $\mathrm{SCI}+$ alendronate $1 \mathrm{mg} / \mathrm{kg}$-treated group decreased significantly compared with the SCI+vehicle-treated group. The level of p53 protein was also significantly reduced (D) compared with the SCI+vehicle group. ${ }^{*} \mathrm{p}<0.05 ;{ }^{* *} \mathrm{p}<0.01$; ${ }^{* * *} \mathrm{p}<0.001$ vs. normal control. ${ }^{*} \mathrm{p}<0.05 ;{ }^{* *} \mathrm{p}<0.01$ vs. SCI+vehicle-treated group. N, normal control; S, sham control; $\mathrm{V}, \mathrm{SCI}+$ vehicle-treated group; $1, \mathrm{SCI}+$ alendronate $1 \mathrm{mg} / \mathrm{kg}$-treated group.

was down-regulated significantly compared with the ratio in the SCI+vehicle-treated group $(3.70 \pm 0.37$ fold and $4.51 \pm 0.92$ fold, respectively; Fig. $4 \mathrm{~A}$ and $4 \mathrm{~B})$. In the $\mathrm{SCI}+$ vehicle-treated group, $\mathrm{p}-$ ERK2/total ERK2 (3.47 \pm 0.20 fold; $\mathrm{p}<0.01)$, $\mathrm{p}$-JNK1/total JNK1 $(2.01 \pm 0.11$ fold; $\mathrm{p}<0.01)$, $\mathrm{p}-\mathrm{JNK} 2 /$ total JNK2 $(4.52 \pm 1.30$ fold; $\mathrm{p}<0.05)$, and $\mathrm{p}-\mathrm{p} 38 /$ total $\mathrm{p} 38(3.27 \pm 0.59$ fold; $\mathrm{p}<0.05)$ were significantly increased at D7PI, the peak stage of inflammation, compared with the ratios in the normal control (Fig. 4A 4C). However, alendronate treatment suppressed the up-regulation of $\mathrm{p}$-JNK1/ total JNK1 $(1.45 \pm 0.16$ fold; $\mathrm{p}<0.01)$ during the same period (Fig. $4 \mathrm{~A} \sim 4 \mathrm{C}$ ). It also significantly suppressed the increase in the level of p53 protein $(1.34 \pm 0.07$ fold and $1.40 \pm 0.15$ fold; $p<0.05$, respectively) at D7PI and D28PI compared to the level in the SCI+vehicletreated group $(1.79 \pm 0.01$ fold and $2.18 \pm 0.21$ fold, respectively; Fig. $4 \mathrm{D})$. The activation of MAPK is implicated in several cellular processes, especially inflammation. The MAPK pathway activation was confirmed as followed by SCI-induced inflammation. This result means that alendronate affects to control the inflammation via MAPK pathway. One of the important roles of MAPK modulates the biosynthesis of pro-inflammatory mediators. Therefore, the pro-inflammatory cytokines and mediators were estimated to affirm that the downstream of MAPK was also changed or not.

\section{Alendronate decreased the secretion of pro-inflammatory mediators}

A quantitative real time-PCR was performed to measure the levels of several pro-inflammatory cytokines and pro-inflammatory mediators at D7PI. The mRNA expression levels of proinflammatory cytokines, including IL-1 $\beta$ (Fig. 5A) and TNF- $\alpha$ (Fig. $5 \mathrm{~B})$ were significantly up-regulated in the $\mathrm{SCI}+$ vehicle-treated group ( $p<0.01$ and $p<0.05$, respectively vs. the normal control). By contrast, alendronate significantly inhibited the up-regulation of 
A

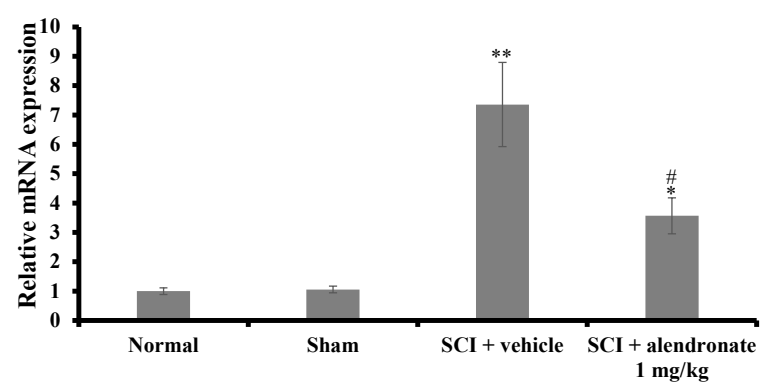

C

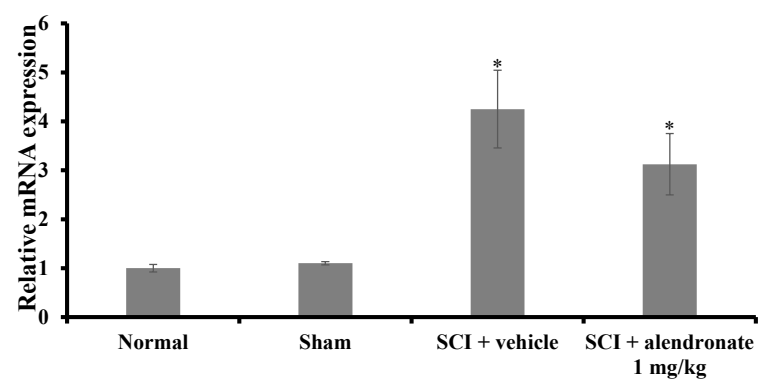

B

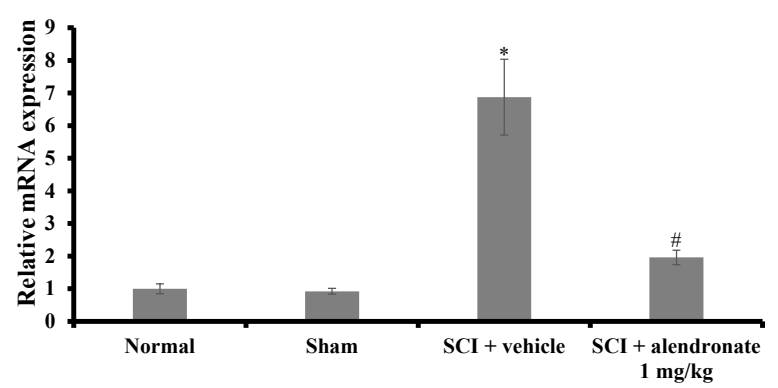

D

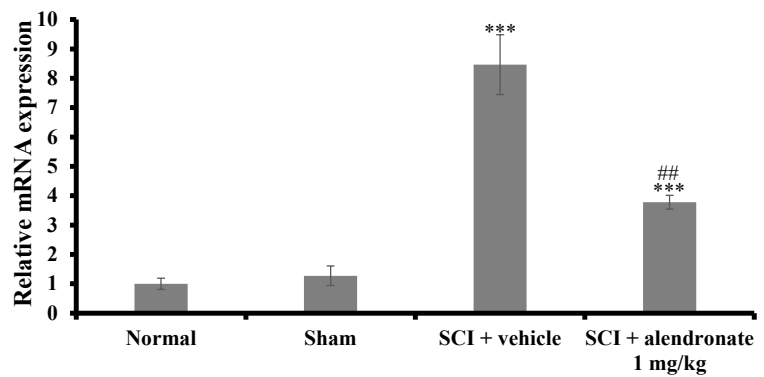

Fig. 5. Analysis of pro-inflammatory responses in normal and SCI-induced rats on D7PI. The levels of the pro-inflammatory cytokines, interleukin-1 beta (IL-1 $\beta)(A)$ and tumor necrosis factor-alpha (TNF- $\alpha)(B)$, increased significantly in the SCI+vehicle-treated group but were significantly reduced by alendronate treatment. Under SCI, the expression levels of the pro-inflammatory mediators, cyclooxygenase-2 (COX-2) (C) and serine peptidase inhibitor, clade A, member $3 \mathrm{~N}$ (Serpina3n) were significantly increased (D). Alendronate treatment did not suppress the increase in the COX-2 mRNA level (C) but it did prevent the up-regulation of Serpina3n expression (D). ${ }^{*} \mathrm{p}<0.05 ;{ }^{* *} \mathrm{p}<0.01 ;{ }^{* * *} \mathrm{p}<0.01$ vs. normal control. ${ }^{*} \mathrm{p}<0.05 ;{ }^{* *} \mathrm{p}<0.01 \mathrm{vs}$. SCI+vehicletreated group.

IL- $1 \beta$ and TNF- $\alpha$ mRNA in the SCI+alendronate $1 \mathrm{mg} / \mathrm{kg}$-treated group ( $\mathrm{p}<0.05$, respectively; Fig. $5 \mathrm{~A}$ and $5 \mathrm{~B}$ ). In addition, the mRNA levels of the pro-inflammatory mediators, cyclooxygenase-2 (COX-2) and Serpina3n, were significantly increased in the SCI+vehicle-treated group $(\mathrm{p}<0.05$ and $\mathrm{p}<0.001$, respectively vs. normal control; Fig. 5C and 5D). However, alendronate treatment did not inhibit the increase in COX-2 mRNA (Fig. 5C) but it did down-regulate the levels of Serpina3n mRNA (Fig. 5D). In accordance with alteration of MAPK pathway as an upstream signaling, the level of pro-inflammatory cytokines and mediators, including IL- $1 \beta$, TNF- $\alpha$ and serpina3n, was changed. These results suggest that the alendronate relieved the inflammatory response via inhibition of MAPK pathway and finally suppressed the synthesis of pro-inflammatory cytokines and mediators.

\section{DISCUSSION}

This is the first study to show that alendronate, a nitrogen-containing bisphosphonate, suppresses the SCI-induced inflammatory responses. SCI can be divided into an early inflammatory stage $(\sim \mathrm{D} 4 \mathrm{PI})$, a cleaning stage (D4PI-D14PI) and a reactive gliosis stage
(D14PI) and includes apoptosis and necrosis [25]. Our results showed that the SCI-induced disability and neuro-inflammation were repressed by alendronate treatment.

Bisphosphonate drugs have been classified as non-nitrogen- and nitrogen-containing bisphosphonates based on their molecular structure [26]. Alendronate is a nitrogen-containing bisphosphonate. The action of nitrogen-containing bisphosphonates involves the mevalonate pathway and inhibition of farnesyl-pyrophosphate synthase [26]. Farnesyl-pyrophosphate synthase suppression modifies GTPase so that the osteoclast cytoskeleton is affected [26]. The effect of alendronate on osteoporosis has been evaluated in ovariectomized rats, where it was shown to promote the healing of osteoporotic fractures [27].

Alendronate improved the learning and memory in a mouse model of Alzheimer's disease by decreasing neuroinflammation, oxidative stress, and the A $\beta 1-42$ deposition induced by an intracerebroventricular streptozotocin infusion [28]. The improvement in Alzheimer's disease in response to alendronate has been shown to result from a significant decrease in $A \beta 1-42$ and $\beta$-site amyloid precursor protein cleaving enzyme-1, a key marker of Alzheimer's disease [28]. In neurodegenerative disease, such as experimental 
autoimmune encephalomyelitis, alendronate mitigates hind-limb paralysis, suppresses $\mathrm{T}$ cell proliferation, and limits the inflammatory response [17]. The treatment with alendronate, as an antiinflammatory molecule, is more effective to diminish the SCIinduced secondary degeneration rather than the pre-treatment of alendronate for preventive effect. Because the minimizing of the secondary degeneration is one of the important matter after SCI. The duration of treatment was depended on the central nervous system insults inflammatory pathology [29]. Unlike the general wound healing process, the infiltration of inflammatory cells in central nervous system insults was persisted from the initiation of injury to few weeks. Based on the characteristic of central nervous system injury, we decided to evaluate the therapeutic effect of alendronate after SCI up to D28PI. Therefore, the alendronate was started to treat after the SCI for D28PI and this result revealed that alendronate ameliorated the severe inflammation in SCI rats.

As mediators of the inflammatory response, the levels of MAPK signaling, p53, IL- $1 \beta$, TNF- $\alpha$, COX-2 and Serpina3n were determined. The key molecules in MAPK signaling are ERK1/2, JNK1/2 and p38 [11]. MAPK signaling activation, via phosphorylation [30], contributes to central nervous system inflammation [31]. A previous study showed that the inhibition of MAPK signaling improved the behavioral dysfunction induced by SCI, by blocking apoptosis [11]. In a rat model of osteoporosis, alendronate reduced the number of apoptotic cells and inflammation by targeting adenylyl cyclase isoform 6, through the regulation of MAPK signaling, including ERK and p38 [32]. MAPK signaling inhibition also alleviates inflammation via the down-regulation of pro-inflammatory cytokines, including IL- $1 \beta$ and TNF- $\alpha$ [33]. Phosphorylated $\mathrm{p} 38$ plays a critical role in the activation of $\mathrm{p} 53$ provoked by chemotherapy [34]. The blockade of p38 inhibits the transcriptional activity of p53 by negatively regulating cell proliferation and mediating the death of highly damaged cells [35]. The increased p38 sequentially promotes p53 [34] and pro-inflammatory cytokines, IL-1 $\beta$ and TNF- $\alpha$ [36]. In our study, p53 and p38 expression increased during the early inflammatory stage, with significant differences between the SCI+vehicle-treated group and the SCI+alendronate $1 \mathrm{mg} / \mathrm{kg}$ treated group on D7PI. These results provided further evidence that alendronate treatment alleviates inflammation after SCI via down-regulation of MAPK signaling and p53.

Serpina $3 n$ is controversially applied as both therapeutic and detrimental implication [19]. Serpina3n has a therapeutic effect on abdominal aortic aneurysm, muscular dystrophy and autoimmune graft rejection, whereas serpina3n is harmful in dilated myocarditis, cancer, Alzheimer's disease and osteoporosis [19]. The escalated serpina3n was identified in the cerebrospinal fluid [37] and was associated with apolipoprotein $\mathrm{E}$ via lipoprotein receptor- related protein pathway, which was leading to aggravation of neurodegenerative response in Alzheimer's disease [38]. The upregulation of serpina3n was detected in reactive astrocyte [21] and reactive astrocyte was involved in promoting or inhibiting axon regeneration $[39,40]$ and anti-oxidant defenses [41]. Reactive astrogliosis is positive response under ischemic injury like stroke, but is negative response in neuroinflammation condition [21]. Additionally, overexpression of serpina3n was detected in various nervous diseases, including high-fat-diet-induced hypothalamic neuro-inflammation [42], trimethyltin chloride-induced neurotoxicity [43] and amyotrophic lateral sclerosis [44]. In case of osteoporosis treated by alendronate, the increased serpina $3 \mathrm{n}$ affected the differentiation of osteoblasts by suppressing alkaline phosphatase activity, osteocalcin and collagen type-1 [45]. In this study, alendronate was confirmed as a potential candidate to regulate the overexpression of serpina3n induced by SCI and relief of SCI-induced neuro-inflammation.

In conclusion, our study showed that the anti-inflammatory properties of alendronate in an animal model of SCI include the reduced infiltration of inflammatory cells and an alleviation of inflammatory responses, including microgliosis/astrogliosis. Based on its demonstrated effects in rats, alendronate treatment may contribute to the functional recovery of SCI in humans.

\section{REFERENCES}

1. Heo SD, Kim J, Choi Y, Ekanayake P, Ahn M, Shin T (2020) Hesperidin improves motor disability in rat spinal cord injury through anti-inflammatory and antioxidant mechanism via Nrf-2/HO-1 pathway. Neurosci Lett 715:134619.

2. Ahmed RU, Alam M, Zheng YP (2019) Experimental spinal cord injury and behavioral tests in laboratory rats. Heliyon 5:e01324.

3. Kjell J, Olson L (2016) Rat models of spinal cord injury: from pathology to potential therapies. Dis Model Mech 9:11251137.

4. Basso DM, Beattie MS, Bresnahan JC (1995) A sensitive and reliable locomotor rating scale for open field testing in rats. J Neurotrauma 12:1-21.

5. Gonzalez-Cano R, Boivin B, Bullock D, Cornelissen L, Andrews N, Costigan M (2018) Up-down reader: an open source program for efficiently processing 50\% von Frey thresholds. Front Pharmacol 9:433.

6. Gale K, Kerasidis H, Wrathall JR (1985) Spinal cord contusion in the rat: behavioral analysis of functional neurologic impairment. Exp Neurol 88:123-134.

7. Deuis JR, Dvorakova LS, Vetter I (2017) Methods used to 
evaluate pain behaviors in rodents. Front Mol Neurosci 10:284.

8. Metz GA, Whishaw IQ (2002) Cortical and subcortical lesions impair skilled walking in the ladder rung walking test: a new task to evaluate fore- and hindlimb stepping, placing, and co-ordination. J Neurosci Methods 115:169-179.

9. Kim DH, Heo SD, Ahn MJ, Sim KB, Shin TK (2003) Activation of embryonic intermediate filaments contributes to glial scar formation after spinal cord injury in rats. J Vet Sci 4:109112.

10. Gwak YS, Kang J, Unabia GC, Hulsebosch CE (2012) Spatial and temporal activation of spinal glial cells: role of gliopathy in central neuropathic pain following spinal cord injury in rats. Exp Neurol 234:362-372.

11. Martini AC, Forner S, Koepp J, Rae GA (2016) Inhibition of spinal c-Jun-NH2-terminal kinase (JNK) improves locomotor activity of spinal cord injured rats. Neurosci Lett 621:5461.

12. Griffin JM, Bradke F (2020) Therapeutic repair for spinal cord injury: combinatory approaches to address a multifaceted problem. ЕMBO Mol Med 12:e11505.

13. Oliveira JR, Oliveira MF (2016) Primary brain calcification in patients undergoing treatment with the biphosphanate alendronate. Sci Rep 6:22961.

14. Sharpe M, Noble S, Spencer CM (2001) Alendronate: an update of its use in osteoporosis. Drugs 61:999-1039.

15. Tricarico PM, Girardelli M, Kleiner G, Knowles A, Valencic E, Crovella S, Marcuzzi A (2015) Alendronate, a doubleedged sword acting in the mevalonate pathway. Mol Med Rep 12:4238-4242.

16. Nishii T, Tamura S, Shiomi T, Yoshikawa H, Sugano N (2013) Alendronate treatment for hip osteoarthritis: prospective randomized 2-year trial. Clin Rheumatol 32:1759-1766.

17. Jung K, Kim J, Ahn G, Matsuda H, Akane T, Ahn M, Shin T (2020) Alendronate alleviates the symptoms of experimental autoimmune encephalomyelitis. Int Immunopharmacol 84:106534.

18. Horvath AJ, Forsyth SL, Coughlin PB (2004) Expression patterns of murine antichymotrypsin-like genes reflect evolutionary divergence at the Serpina3 locus. J Mol Evol 59:488497.

19. Aslam MS, Yuan L (2020) Serpina3n: potential drug and challenges, mini review. J Drug Target 28:368-378.

20. Abraham CR (2001) Reactive astrocytes and alpha1-antichymotrypsin in Alzheimer's disease. Neurobiol Aging 22:931936.

21. Zamanian JL, Xu L, Foo LC, Nouri N, Zhou L, Giffard RG,
Barres BA (2012) Genomic analysis of reactive astrogliosis. J Neurosci 32:6391-6410.

22. Ahn M, Lee C, Jung K, Kim H, Moon C, Sim KB, Shin T (2012) Immunohistochemical study of arginase-1 in the spinal cords of rats with clip compression injury. Brain Res 1445:11-19.

23. Yao Y, Tan YH, Light AR, Mao J, Yu AC, Fu KY (2016) Alendronate attenuates spinal microglial activation and neuropathic pain. J Pain 17:889-903.

24. Choi Y, Oh H, Ahn M, Kang T, Chun J, Shin T, Kim J (2020) Immunohistochemical analysis of periostin in the hearts of Lewis rats with experimental autoimmune myocarditis. J Vet Med Sci 82:1545-1550.

25. Shin T, Ahn M, Moon C, Kim S, Sim KB (2013) Alternatively activated macrophages in spinal cord injury and remission: another mechanism for repair? Mol Neurobiol 47:1011-1019.

26. Panagiotakou A, Yavropoulou M, Nasiri-Ansari N, Makras P, Basdra EK, Papavassiliou AG, Kassi EN (2020) Extra-skeletal effects of bisphosphonates. Metabolism 110:154264.

27. Zhang C, Zhu J, Jia J, Guan Z, Sun T, Zhang W, Yuan W, Wang H, Leng H, Song C (2021) Once-weekly parathyroid hormone combined with ongoing long-term alendronate treatment promotes osteoporotic fracture healing in ovariectomized rats. J Orthop Res 39:2103-2115.

28. Zameer S, Kaundal M, Vohora D, Ali J, Kalam Najmi A, Akhtar M (2019) Ameliorative effect of alendronate against intracerebroventricular streptozotocin induced alteration in neurobehavioral, neuroinflammation and biochemical parameters with emphasis on A $\beta$ and BACE-1. Neurotoxicology 70:122-134.

29. Shechter R, Schwartz M (2013) CNS sterile injury: just another wound healing? Trends Mol Med 19:135-143.

30. Cargnello M, Roux PP (2011) Activation and function of the MAPKs and their substrates, the MAPK-activated protein kinases. Microbiol Mol Biol Rev 75:50-83. Erratum in: Microbiol Mol Biol Rev 2012;76:496.

31. Kaminska B, Gozdz A, Zawadzka M, Ellert-Miklaszewska A, Lipko M (2009) MAPK signal transduction underlying brain inflammation and gliosis as therapeutic target. Anat Rec (Hoboken) 292:1902-1913.

32. Pan BL, Tong ZW, Li SD, Wu L, Liao JL, Yang YX, Li HH, Dai YJ, Li JE, Pan L (2018) Decreased microRNA-182-5p helps alendronate promote osteoblast proliferation and differentiation in osteoporosis via the Rap1/MAPK pathway. Biosci Rep 38:BSR20180696.

33. Bachstetter AD, Van Eldik LJ (2010) The p38 MAP kinase family as regulators of proinflammatory cytokine production in degenerative diseases of the CNS. Aging Dis 1:199-211. 
34. Sanchez-Prieto R, Rojas JM, Taya Y, Gutkind JS (2000) A role for the p38 mitogen-acitvated protein kinase pathway in the transcriptional activation of p53 on genotoxic stress by chemotherapeutic agents. Cancer Res 60:2464-2472.

35. Floriddia EM, Rathore KI, Tedeschi A, Quadrato G, Wuttke A, Lueckmann JM, Kigerl KA, Popovich PG, Di Giovanni $S$ (2012) p53 Regulates the neuronal intrinsic and extrinsic responses affecting the recovery of motor function following spinal cord injury. J Neurosci 32:13956-13970.

36. Kasuya Y, Umezawa H, Hatano M (2018) Stress-activated protein kinases in spinal cord injury: focus on roles of p38. Int J Mol Sci 19:867.

37. Porcellini E, Davis EJ, Chiappelli M, Ianni E, Di Stefano G, Forti P, Ravaglia G, Licastro F (2008) Elevated plasma levels of alpha-1-anti-chymotrypsin in age-related cognitive decline and Alzheimer's disease: a potential therapeutic target. Curr Pharm Des 14:2659-2664.

38. Licastro F, Campbell IL, Kincaid C, Veinbergs I, Van Uden E, Rockenstein E, Mallory M, Gilbert JR, Masliah E (1999) A role for apoE in regulating the levels of alpha-1-antichymotrypsin in the aging mouse brain and in Alzheimer's disease. Am J Pathol 155:869-875.

39. Faulkner JR, Herrmann JE, Woo MJ, Tansey KE, Doan NB, Sofroniew MV (2004) Reactive astrocytes protect tissue and preserve function after spinal cord injury. J Neurosci 24:21432155.

40. Bush TG, Puvanachandra N, Horner CH, Polito A, Ostenfeld T, Svendsen CN, Mucke L, Johnson MH, Sofroniew MV (1999) Leukocyte infiltration, neuronal degeneration, and neurite outgrowth after ablation of scar-forming, reactive astrocytes in adult transgenic mice. Neuron 23:297-308.

41. Dringen R, Gutterer JM, Hirrlinger J (2000) Glutathione metabolism in brain metabolic interaction between astrocytes and neurons in the defense against reactive oxygen species. Eur J Biochem 267:4912-4916.

42. Sergi D, Campbell FM, Grant C, Morris AC, Bachmair EM, Koch C, McLean FH, Muller A, Hoggard N, de Roos B, Porteiro B, Boekschoten MV, McGillicuddy FC, Kahn D, Nicol P, Benzler J, Mayer CD, Drew JE, Roche HM, Muller M, Nogueiras R, Dieguez C, Tups A, Williams LM (2018) SerpinA3N is a novel hypothalamic gene upregulated by a high-fat diet and leptin in mice. Genes Nutr 13:28.

43. Xi Y, Liu M, Xu S, Hong H, Chen M, Tian L, Xie J, Deng P, Zhou C, Zhang L, He M, Chen C, Lu Y, Reiter RJ, Yu Z, Pi H, Zhou Z (2019) Inhibition of SERPINA3N-dependent neuroinflammation is essential for melatonin to ameliorate trimethyltin chloride-induced neurotoxicity. J Pineal Res 67:e12596

44. Saris CG, Groen EJ, Koekkoek JA, Veldink JH, van den Berg LH (2013) Meta-analysis of gene expression profiling in amyotrophic lateral sclerosis: a comparison between transgenic mouse models and human patients. Amyotroph Lateral Scler Frontotemporal Degener 14:177-189.

45. Ishida M, Kawao N, Okada K, Tatsumi K, Sakai K, Nishio K, Kaji H (2018) Serpina3n, dominantly expressed in female osteoblasts, suppresses the phenotypes of differentiated osteoblasts in mice. Endocrinology 159:3775-3790. 